\title{
The Cholesterol-Dependent Cytolysin Signature Motif: A Critical Element in the Allosteric Pathway that Couples Membrane Binding to Pore Assembly
}

\author{
Kelley J. Dowd, Rodney K. Tweten*
}

Department of Microbiology and Immunology, The University of Oklahoma Health Sciences Center, Oklahoma City, Oklahoma, United States of America

\begin{abstract}
The cholesterol-dependent cytolysins (CDCs) constitute a family of pore-forming toxins that contribute to the pathogenesis of a large number of Gram-positive bacterial pathogens.The most highly conserved region in the primary structure of the CDCs is the signature undecapeptide sequence (ECTGLAWEWWR). The CDC pore forming mechanism is highly sensitive to changes in its structure, yet its contribution to the molecular mechanism of the CDCs has remained enigmatic. Using a combination of fluorescence spectroscopic methods we provide evidence that shows the undecapeptide motif of the archetype CDC, perfringolysin O (PFO), is a key structural element in the allosteric coupling of the cholesterol-mediated membrane binding in domain 4 (D4) to distal structural changes in domain 3 (D3) that are required for the formation of the oligomeric pore complex. Loss of the undecapeptide function prevents all measurable D3 structural transitions, the intermolecular interaction of membrane bound monomers and the assembly of the oligomeric pore complex. We further show that this pathway does not exist in intermedilysin (ILY), a CDC that exhibits a divergent undecapeptide and that has evolved to use human CD59 rather than cholesterol as its receptor. These studies show for the first time that the undecapeptide of the cholesterol-binding CDCs forms a critical element of the allosteric pathway that controls the assembly of the pore complex.
\end{abstract}

Citation: Dowd KJ, Tweten RK (2012) The Cholesterol-Dependent Cytolysin Signature Motif: A Critical Element in the Allosteric Pathway that Couples Membrane Binding to Pore Assembly. PLoS Pathog 8(7): e1002787. doi:10.1371/journal.ppat.1002787

Editor: Theresa M. Koehler, The University of Texas-Houston Medical School, United States of America

Received April 6, 2012; Accepted May 19, 2012; Published July 5, 2012

Copyright: (C) 2012 Dowd, Tweten. This is an open-access article distributed under the terms of the Creative Commons Attribution License, which permits unrestricted use, distribution, and reproduction in any medium, provided the original author and source are credited.

Funding: These studies were supported by a grant to RKT from the National Institutes of Health, NIAID (AI037657). The funders had no role in study design, data collection and analysis, decision to publish, or preparation of the manuscript.

Competing Interests: The authors have declared that no competing interests exist.

* E-mail: Rodney-Tweten@ouhsc.edu

\section{Introduction}

The cholesterol-dependent-cytolysin (GDC) family of toxins consists of over 25 members that are produced by many different species of Gram-positive bacterial pathogens [1] and contribute in various ways to the pathogenesis of these organisms $[2,3,4,5]$. Members of this family exhibit high levels of homology in their primary structures $(40-70 \%)$ and in the crystal structures of their soluble monomers $[6,7,8,9]$. The region within the CDC primary structure that exhibits the highest degree of sequence identity is an 11-residue peptide known as the undecapeptide or tryptophan-rich motif, which is located near the C-terminus of the molecule in domain 4 (D4) (Fig. 1). The undecapeptide (ECTGLAWEWWR) is the signature motif for the CDCs [1] and so proteins exhibiting this peptide sequence have a high probability of belonging to the GDC family. The pore forming mechanism of the CDCs that use cholesterol as their receptoris highly sensitive to changes in the primary structure of the undecapeptide $[6,10,11,12,13,14,15]$. These studies suggest that the undecapeptide plays an important role in the CDC poreforming mechanism, yet since Iwamoto et al. [16] began studying the effects of chemically altering the undecapeptide in 1987 its contribution to the pore forming mechanism of the GDGs has remained elusive.

The undecapeptide is located at the tip of D4 of the CDC structure, as shown in the structure of the CDC produced by
Clostridium perfringens, perfringolysin O (PFO) (Fig. 1). D4 also contains the cholesterol recognition/binding motif (CRM) and two other short loops (L2 and L3) near the undecapeptide (reviewed in [17]). Upon recognition of membrane cholesterol by the CRM,loops L2 and L3 insert into the membrane. These interactions anchor the monomers in a perpendicular orientationto the membrane surface where the tip of $\mathrm{D} 4$ is anchored to the membrane surface and the top of D 3 resides about $113 \AA$ above the membrane surface $[18,19,20]$. Although the sidechains of several residues of loops L2 and L3 and the undecapeptide insert into and anchor the monomers to the membrane they do not penetrate deeply into the bilayer core [19,21].

It had been generally accepted in the field that the undecapeptide motif wasthe CRM of the GDCs, although this function had never been demonstrated unambiguously. An early study by Iwamoto et al. [16] showed that chemical modification of the undecapeptide cysteine caused independent defects in both binding and pore formation. Since that time it has been shown that mutation of many of the undecapeptide residues often affects both binding and pore formation [6,10,11,12,13,14,15]. We recently showed, however, that the CRMresides in the nearby D4 loop L1 (Fig. 1) and is comprised of a threonine-leucine pair that is strictly conserved in all known CDCs [22]. Upon cholesterol binding by the CRM the nearby loops L2 and L3 and the conserved undecapeptide insert into the bilayer surface 


\section{Author Summary}

The CDCs are a large family of pathogenesis-associated pore-forming toxins that are expressed by many Grampositive pathogens. The conserved undecapeptide motif of the CDCs has been regarded as the signature peptide sequence for these toxins, yet its function has remained obscure. The studies herein show that the undecapeptide forms a critical structural element in the allosteric pathway that couples membrane binding to cholesterol to the initiation of distal structural changes, which are required for the assembly of the pore forming complex. These studies provide the first insight into the function of this highly conserved sequence and show that through evolution this pathway is missing in the CD59-binding CDCs.

and anchor the monomer in a perpendicular orientation to the membrane surface $[19,21,23,24]$. Membrane binding in conjunction with monomer-monomer interactions [20] initiates and drives a dramatic series of secondary and tertiary structural changes in D3, which is about $60 \AA$ distant from the tip of D4 (Fig. 1). These structural changes are necessary for the assembly of the membrane bound monomers into the large oligomeric pore complex $[23,24,25,26,27]$. Soluble monomers of PFO do not exhibit these D3 structural changes, even at the high concentrations required for crystallization of the protein [28]: membrane binding is required to initiate the structural changes in D3 [20,25].

As indicated above, the pore-forming mechanism of PFO-like CDCs is highly sensitive to mutations in the undecapeptide $[6,10,11,12,13,14,15]$. Furthermore, the conformational changes in the PFO undecapeptide, reflected by the membrane insertion of its tryptophan residues, are conformationally coupled to the structural changes in TMH1 required for the formation of the $\beta$ barrel pore [20]. This observation suggests that the undecapeptide of PFO is involved in the allosteric coupling of membrane binding to the initiation of the D3 structural changes that are necessary for monomer-monomer interaction and the formation of the oligomeric $\beta$-barrel pore complex.

A small family of CDCs, typified by Streptococcus intermedius intermedilysin (ILY) use human CD59 as their receptor, rather than cholesterol [29,30,31]. The D3 structural changes in ILY can be initiated by binding to human CD59 in membranes that are largely, though not completely depleted of cholesterol [32]. ILY still requires a CRM-mediated membrane interaction with cholesterol to maintain its anchor to the membrane surface (it disengages from CD59 during prepore to pore conversion $[22,33])$, but it remains unclear if cholesterol binding also participates in initiation of the D3 structural changes necessary for assembly of the oligomer pore complex. Interestingly, in contrast to the CDCs that use cholesterol as their receptor, the pore forming mechanism of ILY is comparatively insensitive to mutations within its undecapeptide [6], which suggests that it may not play as significant of a role in the pore forming mechanism of these toxins.

In the present study we performed a detailed molecular analysis of a point mutation in the undecapeptide of PFO that reduces its pore-forming activity 100-fold, whereasthe analogous mutation has no significant effect on the mechanism of ILY [6]. In PFO this mutant blocks all measurable structural transitions in D3 and prevents the stable interaction of membrane-bound monomers. We further show that the effect of this mutation on the activity of PFO is similar to that observed for cholesterol bound native ILY in the absence of CD59. These results show that the undecapeptide of PFO is a critical structure within the allosteric pathway of PFO that couples cholesterol binding to the initiation of structural changes within D3, which lead to the formation of the $\beta$-barrel pore. We further show that this pathway appears to be missing in the CD59-binding ILY, so that assembly of its pore complex is initiated by its interaction with CD59 rather than cholesterol.

\section{Results}

\section{Cytolytic activity of PFO mutated at Arg-468}

Arg-468 is the last residue of the PFO undecapeptide (ECTGLAWEWWR), as well as in the ILY undecapeptide (GATGLAWEPW $\underline{\mathbf{R}}$. Substitution of the PFO undecapeptide at this residue with alanine decreases its hemolytic activity 100 -fold (Table 1), whereas substitution of the analogous residue in ILY has little effect on the activity [6]. A series of mutants were generated for Arg-468 of PFO to examine the effects of size, length and charge of the residue atposition 468 on the hemolytic activity of PFO (Table 1). Neither conservative nor non-conservative substitutions were tolerated: all substitutions decreased hemolytic activity $\geq 100$-fold. Based on the crystal structure of PFO the only intramolecular contacts established by Arg-468 are hydrogen bonds between its sidechain $\mathrm{NH} 1$ and the CRM carbonyls (Fig. 1B). Interestingly, this contact is lost in the ILY monomer (Fig. 1G), which presumably results from differences in its undecapeptide structure [6]. We selected the $\mathrm{PFO}^{\mathrm{R} 468 \mathrm{~A}}$ mutant for further studies into the defect(s) induced by substitution of the Arg-468 residue on the PFO pore-forming mechanism.

\section{$\mathrm{PFO}^{\mathrm{R} 468 \mathrm{~A}}$ membrane binding}

We have shown that a conserved Thr-Leu pair in Loop 1, and not the undecapeptide, is responsible for CDC binding to membrane cholesterol [22], yet mutations within the conserved undecapeptide were often observed to affect binding [6,13,34]. To confirm that the loss of hemolytic activity by the PFO ${ }^{\mathrm{R} 468 \mathrm{~A}}$ mutant was not due solely to a defect in binding we examined the ability of the mutant to bind to human RBCs by flow cytometry. In order to prevent cell lysis at high concentrations of toxin, derivatives of native $\mathrm{PFO}$ and $\mathrm{PFO}^{\mathrm{R} 468 \mathrm{~A}}$ were generatedin which an engineered disulfide was introduced between residuesThr-319 in $\beta 4$ and Val334 in $\beta 5$ that prevent the rotation of $\beta 5$ away from $\beta 4$ in domain $3\left(\mathrm{PFO}^{\beta 4 \beta 5}\right.$ and $\left.\mathrm{PFO}^{\mathrm{R} 468 \mathrm{~A} \bullet \beta 4 \beta 5}\right)$. The engineered disulfide thereforepreventsthe formation of a functional pore by blocking the intermolecular interaction of $\beta 1$ of one monomer with $\beta 4$ of another monomer [25]. A third cysteine was substituted at residue Asp-30 in both mutants, which is at the amino terminus of PFO, so that specific fluorescent probes could be introduced into these

Table 1. Cytolytic activity of PFO derivatives with mutations in the Arg-468 residue.

\begin{tabular}{lll}
\hline Toxin & EC $_{\mathbf{5 0}}(\mathbf{M})$ & \% WT \\
\hline PFO & $6.0 \times 10^{-11}$ & 100 \\
R468A & $6.0 \times 10^{-9}$ & 1 \\
R468K & $5.0 \times 10^{-9}$ & 1 \\
R468Q & $2.4 \times 10^{-8}$ & $<1$ \\
R468E & $3.9 \times 10^{-8}$ & $<1$ \\
\hline
\end{tabular}

Cytolytic activity of PFO and Arg-468 mutants is shown as the effective concentration $\left(\mathrm{EC}_{50}\right.$ ) of toxin required for $50 \%$ lysis of human erythrocytes under standard assay conditions (see Materials and Methods for details). doi:10.1371/journal.ppat.1002787.t001 


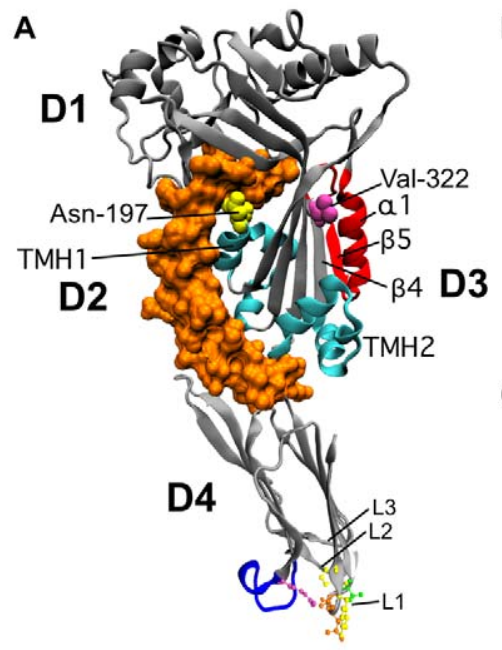

D
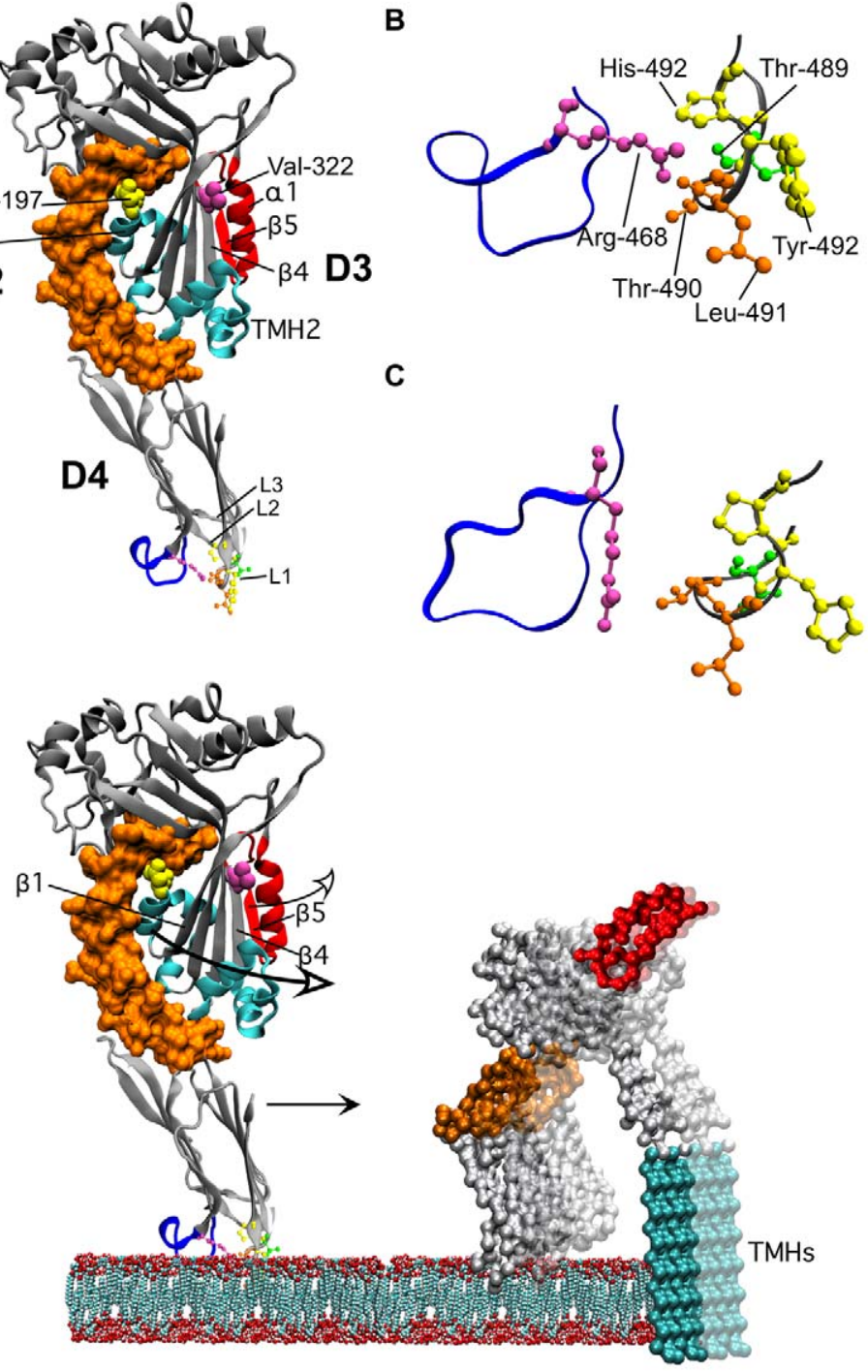

C

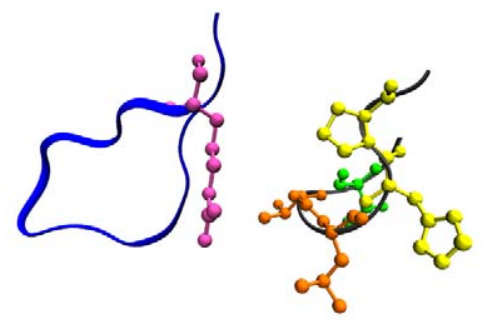

Figure 1. The molecular structures of PFO and ILY. Shown in A is a ribbon representation of the crystal structure of PFO [7]. The domain $3 \beta 5$ strand and associated $\alpha$-helix $(\alpha 1)$ that swing away from $\beta 4$ are highlighted in red. The locations of Asn-197 (at the D2-D3 interface) and Val-322 (buried under the loop formed by $\alpha 1 \beta 5$ ) are shown in space-filled atoms. The twin $\alpha$-helical bundles in D3 (cyan) extend into the twin transmembrane $\beta$-strands (TMHs). The conserved undecapeptide is shown in blue in D4. In panel B an enlarged view of the conserved undecapeptide loop and the CRM containing loop L1 of PFO is shown. In Panel C the analogous structures are shown for ILY as are shown in panel B for PFO.All structures were derived from the crystal structures of PFO and ILY $[6,7])$. In panel D we show the structural changes in PFO as it makes the transition from the bound monomer state to the membrane embedded oligomer. The membrane embedded monomer structure is based on the 3D reconstruction of the pneumolysin pore fitted with the PFO crystal structure [57]. D3 breaks its contacts with D2 and swings out in order to extend the $\alpha$-helical bundles (in cyan) into the twin TMHs. This transition also repositions Asn-197 from the D2-D3 interface to a solvent exposed position within the lumen of the membrane pore. Prior to or simultaneously with the disruption of the D2-D3 interface the $\alpha 1 \beta 5$ loop (red) swings away from $\beta 4$ thus exposing the edge $\beta 4$ (as well as exposing Val-322 to the solvent), which can then pair with $\beta 1$ of a second monomer. Upon transition to the pore the oligomeric complex undergoes a $40 \AA$ vertical collapse to insert the $\beta$-barrel pore into the bilayer [23]. After $\beta 5$ breaks contact with $\beta 4$ the location of $\alpha 1 \beta 5$ loop is not known, its position in the model is for illustrative purposes only. All structures were generated using VMD [58].

doi:10.1371/journal.ppat.1002787.g001

mutants. This mutation does not affectthe structure of PFO or its function [35].

At the highest concentration of $\mathrm{PFO}^{\mathrm{R} 468 \mathrm{~A}}$ we observed about a $50 \%$ decrease in binding to hRBCs compared to PFO, although at lower concentrations this difference was greater (Fig. 2A). The decrease in binding, however, doesn't account for the 100-fold decrease in cytolytic activity. PFO follows an ordered series of coupled conformational changes that are initiated by binding $[19,20,23,25]$, therefore the major defect induced by the $\mathrm{PFO}^{\mathrm{R} 468 \mathrm{~A}}$ mutation affects an event after binding, which then prevents formation of the pore complex.

\section{Oligomerization of PFO ${ }^{\mathrm{R} 468 \mathrm{~A}}$}

Upon membrane binding PFO monomers oligomerize into large SDS-resistant prepore complexes containing approximately 36 monomers [23]. An oligomerization assay was performed with the $\mathrm{PFO}^{\mathrm{R} 468 \mathrm{~A}}$ mutant. Due to the lower binding affinity observed in Fig. 2A we increased the concentration of human red blood cells (hRBCs) to ensure complete binding of $\mathrm{PFO}^{\mathrm{R} 468 \mathrm{~A}}$. The concentration of hRBCs in the binding studies in Fig. 2A was maintained at $4 \times 10^{6} / \mathrm{ml}$ whereas in the oligomerization assay shown in Fig. 2B the concentration hRBCs ranged from $2.5 \times 10^{7}$ to 
A

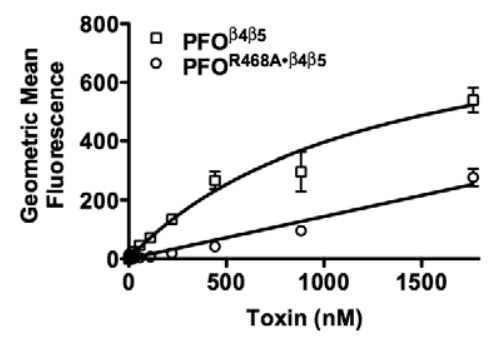

B

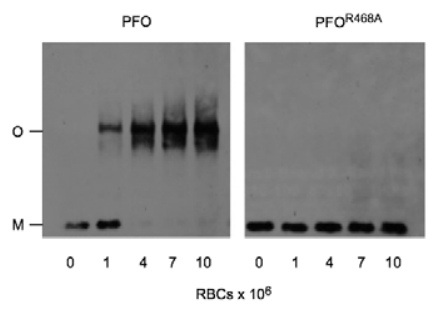

Figure 2. Binding and oligomerization of $\mathrm{PFO}$ and $\mathrm{PFO}^{\mathbf{R 4 6 8 A}}$. (A) Binding of $\mathrm{PFO}^{\beta 4 \beta 5}$ and $\mathrm{PFO} \mathrm{O}^{\mathrm{R} 468 \mathrm{~A} \cdot \beta 4 \beta 5}$ to human $\mathrm{RBCs}\left(4 \times 10^{6} / \mathrm{ml}\right.$ in a final volume of $0.5 \mathrm{ml}$ ) was measured by flow cytometry. The disulfide locked $\beta 4 \beta 5$ versions of each protein [25] were used to prevent the lysis of the RBCs during flow cytometry. (B). Oligomerization of $\mathrm{PFO}$ and $\mathrm{PFO}^{\mathrm{R} 468 \mathrm{~A}}$ (both toxins were maintained at $440 \mathrm{nM}$ )on human RBCs (concentrations ranged from $2.5 \times 10^{7} / \mathrm{ml}$ to $2.5 \times 10^{8} / \mathrm{ml}$ in a final volume of $40 \mu \mathrm{l}$ ) was determined using SDS-agarose gel electrophoresis (SDS-AGE) and the proteins were detected with anti-PFO antibody after transfer to nitrocellulose paper. The analyses are representative of at least 3 experiments. doi:10.1371/journal.ppat.1002787.g002

$2.5 \times 10^{8} / \mathrm{ml}$, which wasapproximately $6-60$ fold higher than the concentration used in the flow cytometry assay. After the toxins were allowed to bind, the samples were solubilized with SDS without heating and separated by SDS-agarose gel electrophoresis (SDS-AGE), which separates the monomer and oligomer forms [36]. Native PFO readily formed SDS-resistant oligomers at all concentrations of RBCs (Fig. 2B) whereas $\mathrm{PFO}^{\mathrm{R} 468 \mathrm{~A}}$ did not form detectable levels of SDS-resistant oligomers (Fig. 2B). Therefore, the major defect in the $\mathrm{PFO}^{\mathrm{R} 468 \mathrm{~A}}$ pore-forming mechanism follows binding and prevents the formation of an SDS-stable oligomer.

\section{Rotation of $\beta 5$ away from $\beta 4$}

Several structural transitions in domain 3 are initiated by membrane binding, which are required for oligomerization and pore formation $[25,26,27]$. One of these structural transitions is the rotation of $\beta$-strand 5 ( $\beta 5$ ) away from the adjacent $\beta$-strand 4 $(\beta 4)$ of the core $\beta$-sheet in domain 3 (Fig. 1A), whichcontributes to the formation of the SDS-resistant prepore oligomer [25]. Rotation of $\beta 5$ away from $\beta 4$ allows the formation of edge-on hydrogen bonds between the peptide backbones of $\beta 4$ and $\beta 1$ of two membrane-bound monomers [25].

The disruption of the $\beta 4 / \beta 5$ interaction can be followed spectroscopically using the environmentally sensitive fluorescent probe, NBD (7-nitrobenz-2-oza-1,3 diazole) [27], which is positioned on the sulfhydryl of a cysteine substituted for Val-322 in $\beta 4$ (Fig. 1A). Val-322 is buried under the residues of $\beta 5$ and so a probe positioned here is in a hydrophobic pocket. The fluorescence emission of NBD is quenched by water, therefore as $\beta 5$ rotates away from $\beta 4$ the NBD positioned in $\beta 4$ moves from a nonpolar to polar environment, which results in a decrease in its fluorescence emission intensity as it is exposed to the aqueous milieu [25]. The $\mathrm{PFO}^{\mathrm{R} 468 \mathrm{~A} \cdot \mathrm{V} 322 \mathrm{C}-\mathrm{NBD}}$ mutant exhibited virtually no change in the NBD emission compared to functional PFO ${ }^{\text {V322C-NBD }}$ (Fig. 3). These results show that the rotation of $\beta 5$ away from $\beta 4$ does not occur in membrane bound $\mathrm{PFO}^{\mathrm{R} 468 \mathrm{~A}}$.

\section{Monomer-monomer interaction of $\mathrm{PFO}^{\mathrm{R} 468 \mathrm{~A}}$}

The studies above show that $\mathrm{PFO}^{\mathrm{R} 468 \mathrm{~A}}$ does not form SDSresistant oligomers, which is likely due to the loss of the intermolecular $\beta 1-\beta 4$ interaction of monomers. This observation, however, did not rule out the possibility that $\mathrm{PFO}^{\mathrm{R} 468 \mathrm{~A}}$ monomers could still form a SDS-sensitive oligomer. To determine whether $\mathrm{PFO}^{\mathrm{R} 468 \mathrm{~A}}$ formed SDS-sensitive oligomers the $\mathrm{PFO}^{\mathrm{R} 468 \mathrm{~A}}$ monomer association was examined using fluorescence resonance energy transfer (FRET). A cysteine was substituted for the amino terminal Asp-30 and labeled with either donor fluorophore (D) (Alexa Fluor 488) or acceptor fluorophore (A) (Alexa Fluor 568). A mixture containing a 4:1 molar ratio of A-labeled $\mathrm{PFO}^{\mathrm{R} 468 \mathrm{~A}}$ or unlabeled $\mathrm{PFO}^{\mathrm{R} 468 \mathrm{~A}}(\mathrm{U})$ to D-labeled toxin was incubated with membranes and fluorescence emission intensity of $\mathrm{D}$ was measured.

When membrane-bound PFO monomers associate to form the prepore oligomer the distance $\left(\mathrm{R}_{0}\right)$ between $\mathrm{D}$ and Afluorescent dyes on the monomers decreases, which results in the FRETdependent quenching of the $\mathrm{D}$ emission $\left(\mathrm{R}_{0}\right.$ is typically $\left.<10 \mathrm{~nm}\right)$ [37]. As expected, we observed an A-dependent quenching of the $\mathrm{D}$ emission for functional PFO as it oligomerized [35,37], whereas no change in the donor fluorescence was observed for $\mathrm{PFO}^{\mathrm{R} 468 \mathrm{~A}}$ (Fig. 4). This result shows that the $\mathrm{PFO}^{\mathrm{R} 468 \mathrm{~A}}$ monomers do not interact, or only form transient interactions that cannot be detected by FRET. FRET requires the donor and acceptor pair be at a fixed distance during the lifetime of the donor emission, which for Alexa-488 is approximately $4 \mathrm{~ns}$ [38]. Therefore the $\mathrm{PFO}^{\mathrm{R} 468 \mathrm{~A}}$ monomers are, at most, only interacting briefly within a timeframe that is shorter than the fluorescence lifetime of the Alexa dye.

\section{The status of the domain $3 \mathrm{TMHs}$ in $\mathrm{PFO}^{\mathrm{R} 468 \mathrm{~A}}$}

The D2-D3 interface is disrupted in order to extend the D3 $\alpha$ helical bundle into transmembrane $\beta$-hairpin 1 (TMH1) [27], which together with TMH2 ultimately contribute to the formation of the membrane spanning $\beta$-barrel pore $[26,27]$. First, the $\alpha$ helical bundle that forms TMH1 must break its interaction with D2 to unravel and form the extended $\beta$-hairpin structure, which eventually inserts into the bilayer as part of the $\beta$-barrel pore [27]. Disruption of the TMH1 contact with D2 can be measured by
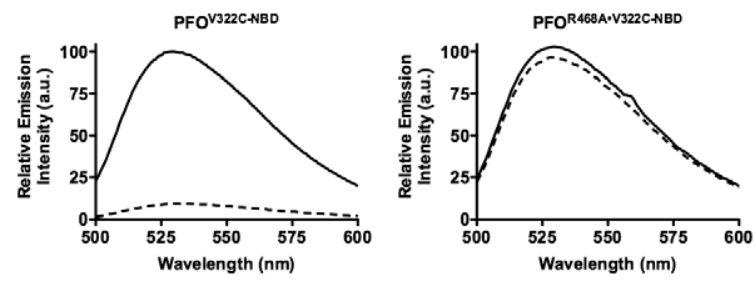

Figure 3. Disruption of the $\beta 4 \beta 5$ interface of PFO and PFO ${ }^{\mathrm{R} 468 \mathrm{~A}}$. A cysteine was substituted for Val-322, located in the D3 $\beta 4$ strand. Each derivative was labeled with NBD and incubated in the presence (dashed line) and absence (solid line) of human erythrocyte ghost membranes. The fluorescence emission intensity of NBD was measured from 500$600 \mathrm{~nm}$. The data are representative of 3 experiments. doi:10.1371/journal.ppat.1002787.g003 

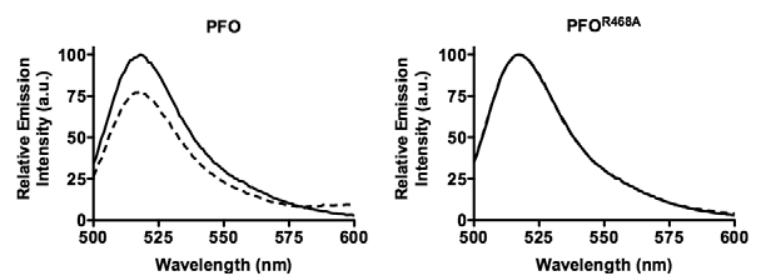

Figure 4. FRET-detected monomer association of PFO and PFO $^{\mathbf{R 4 6 8 A}}$. A cysteine was substituted for Asp-30, located in domain 1 and the derivatives were labeled with Alexa Fluor 488 (donor, D) or Alexa Fluor 568 (acceptor, A).A 4:1 molar ratio of A-labeled PFO ${ }^{\text {R468A }}$ (dashed line) or unlabeled PFO ${ }^{\mathrm{R} 468 \mathrm{~A}}$ ( $\mathrm{U}$; solid line) to $\mathrm{D}$-labeled toxin was incubated in the presence of human erythrocyte ghost membranes and fluorescence emission intensity of $D$ was measured.

doi:10.1371/journal.ppat.1002787.g004

placing a NBD probe on a cysteine substituted for Asn197inTMH1 (Fig. 1). Asn-197 resides at this interface and undergoes a nonpolar to polar transition as the $\alpha$-helical bundle breaks contact with D2 and unravels to form the extended $\beta$ hairpin [27]. The subsequent insertion of the $\beta$-barrel pore can be followed by placing a NBD probe on cysteine-substituted Ala-215 in TMH1, which undergoes a polar to nonpolar transition as its sidechain inserts into the bilayer core [27].

As expected, the fluorescence emission of the NBD probe on cysteine substituted Asn-197 in native PFO decreases to less than $25 \%$ of its initial value as the $\alpha$-helical bundle disengages from its interface with D2 (Fig. 5A, left panel). Also, as expected, the fluorescence emission of the NBD probe located at position 215 in TMH1 of PFO increases as it makes the transition from its polar environment in the soluble monomer to its membrane embedded position in the $\beta$-barrel pore (Fig. $6 \mathrm{~A}$, left panel). In contrast, little change was detected in the fluorescence emission of the NBD probe at both locations in membrane bound $\mathrm{PFO}^{\mathrm{R} 468 \mathrm{~A} \cdot \mathrm{N} 197 \mathrm{C}-\mathrm{NBD}}$, showing that TMH1 did not disengage from its interface with $\mathrm{D} 2$ (Fig. 5A, right panel) and insert into the membrane (Fig. 6A, right panel).
Native PFO drives the membrane insertion of the $\mathrm{PFO}^{\mathrm{R} 468 \mathrm{~A}} \beta$-barrel pore

As shown above, the membrane-bound monomers of $\mathrm{PFO}^{\mathrm{R} 468 \mathrm{~A}}$ do not interact and the D3 structural transitions that lead to the insertion of the $\beta$-barrel pore do not occur in $\mathrm{PFO}^{\mathrm{R} 468 \mathrm{~A}}$ : in essence the monomers remain inert after binding. Therefore, we next determined whether functional PFO could form chimeric oligomers with $\mathrm{PFO}^{\mathrm{R} 468 \mathrm{~A}}$ and drive these structural transitions.

The same experiments were performed as in Figs. 5A and 6A except that a 4:1 ratio of unlabeled $\mathrm{PFO}$ or $\mathrm{PFO}^{\mathrm{R} 468 \mathrm{~A}}$ was mixed with the labeled species prior to their addition to the liposomes. As expected, the relative emission intensity of the NBD probe was similar when each fluorescence species was mixed with a 4-molar excess of the unlabeled homologous protein. For PFO N197C-NBD compare the left panels of Fig. 5A and Fig. 5B and for $\mathrm{PFO}^{\mathrm{A} 215 \mathrm{C}-\mathrm{NBD}}$ compare the left panels of Fig. 6A and Fig. 6B. Similarly, no change was observed in the NBD emission for $\mathrm{PFO}^{\mathrm{R} 468 \mathrm{~A} \cdot \mathrm{N} 197 \mathrm{C}-\mathrm{NBD}}$ (compare Fig. 5A, right panel to Fig. 5B center panel) and for PFO ${ }^{\mathrm{R} 468 \mathrm{~A} \cdot \mathrm{A} 215 \mathrm{C}-\mathrm{NBD}}$ (compare Fig. 6A, right panel to Fig. $6 \mathrm{~B}$ center panel) when they were mixed with a 4-molar excess of unlabeled $\mathrm{PFO}^{\mathrm{R} 468 \mathrm{~A}}$.

However, when a 4-molar excess of unlabeled PFO was mixed with the NBD-labeled species of $\mathrm{PFO}^{\mathrm{R} 468 \mathrm{~A}}$ it drove the disruption of the D2-D3 interface and insertion of the $\beta$-barrel pore. We observed the expected decrease in the fluorescence emission of the NBD probe located at the D2-D3 interface (compare the right and left panel of Fig. 5), as $\beta 5$ swings away form $\beta 4$. Also, the relative emission intensity increased as the probe located at position 215 inserted into the bilayer (compare the right and left panels in Fig. 6). Furthermore, the change in the emission intensity of the NBDin $\mathrm{PFO}^{\mathrm{R} 468 \mathrm{~A} \cdot \mathrm{A} 215 \mathrm{C}-\mathrm{NBD}}$ when mixed with a 4-molar excess of PFO was quantitatively similar to that observed for PFO ${ }^{\mathrm{A} 215 \mathrm{C}-\mathrm{NBD}}$ alone or mixed with the unlabeled PFO. Therefore, nearly all of the $\mathrm{PFO}^{\mathrm{R} 468 \mathrm{~A} \bullet \mathrm{A} 215 \mathrm{C}-\mathrm{NBD}} \mathrm{TMHs}$ were converted to a membraneinserted state.

These results show that functional PFO can form sufficient intermolecular contacts with $\mathrm{PFO}^{\mathrm{R} 468 \mathrm{~A}}$ to efficiently drive the

A
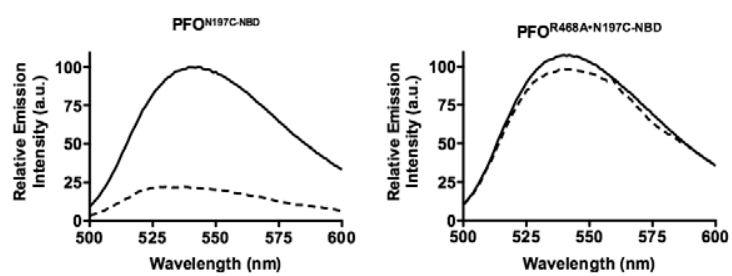

B
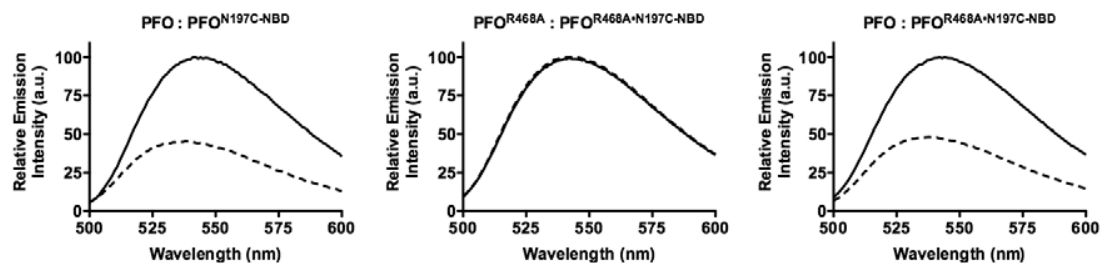

Figure 5. Disruption of the D2/D3 interface in PFO and PFO ${ }^{\text {R468A }}$. (A) A cysteine was substituted for TMH1 residue Asn-197, which is located within the D2/D3 interface. Each derivative was labeled with NBD and incubated in the presence (dashed line) and absence (solid line) of human erythrocyte ghost membranes. If TMH1 breaks its contact with D2 then Asn-197 moves from a buried, nonpolar location at the interface with D2 to the lumen of the pore. An NBD positioned at this location will therefore undergo a nonpolar to polar transition, which results in the quenching of the fluorescence emission. (B) Unlabeled native PFO or PFO ${ }^{\mathrm{R} 468 \mathrm{~A}}$ were mixed at a 4:1 molar ratio with PFO ${ }^{\mathrm{N} 197 \mathrm{C}-\mathrm{NBD}}$ and PFO ${ }^{\mathrm{R} 468 \mathrm{~A} \cdot \mathrm{N} 197 \mathrm{C}-\mathrm{NBD}}$ derivatives. The fluorescence emission intensity of NBD was measured from 500 to $600 \mathrm{~nm}$. These data are representative of 3 experiments. doi:10.1371/journal.ppat.1002787.g005 

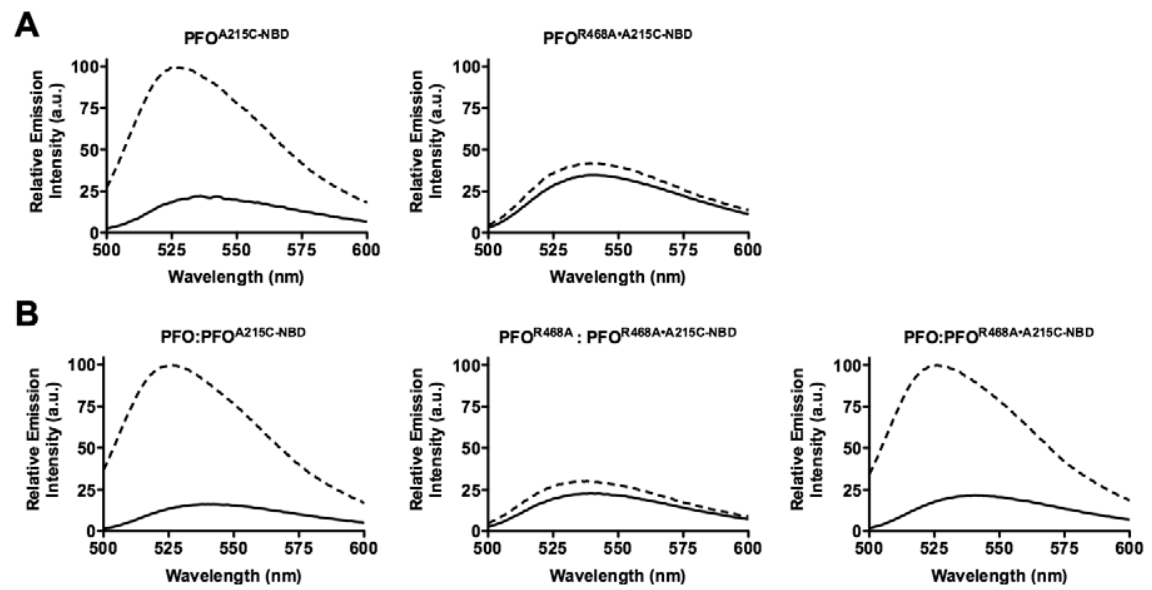

Figure 6. TMH insertion in PFO and $\mathbf{P F O}^{\mathbf{R 4 6 8 A}}$. A cysteine was substituted for Ala-215 in PFO and PFO ${ }^{\mathrm{R} 468 \mathrm{~A}}$, which is located in TMH1.The sidechain of Ala-215 is in an aqueous environment in the soluble monomer, but enters the membrane upon formation of the membrane spanning $\beta$ barrel [27]. Therefore an NBD probe positioned at this site undergoes a polar to nonpolar transition that is detected by an increase in the fluorescence emission of the probe.(A) Each NBD-labeled derivative was incubated in the presence (dashed line) and absence (solid line) of human erythrocyte ghost membranes. (B) Unlabeled native PFO or PFO ${ }^{\mathrm{R} 468 \mathrm{~A}}$ were mixed in a 4:1 molar ratio with PFO ${ }^{\mathrm{A} 215 \mathrm{C}-\mathrm{NBD}}$ and PFO ${ }^{\mathrm{R} 468 \mathrm{~A} \cdot \mathrm{A} 215 \mathrm{C}-\mathrm{NBD}}$ derivatives. The fluorescence emission for all experiments wasrelative to the maximum emission change observed for NBD-labeled PFO. The fluorescence emission intensity of NBD was measured from $500-600 \mathrm{~nm}$. The data are representative of 3 experiments.

doi:10.1371/journal.ppat.1002787.g006

disruption of its domain 2-3 interface and the membrane insertion of its $\beta$-barrel. Hence, $\mathrm{PFO}^{\mathrm{R} 468 \mathrm{~A}}$ is competent to undergo the necessary D3 structural changes and insert its $\mathrm{TMHs}$ into the membrane, but is unable to initiate these changes because it is missing the allosteric signal that is initiated by membrane binding. These data also indicate that the rate of binding of the $\mathrm{PFO}^{\mathrm{R} 468 \mathrm{~A}}$ monomers to the membrane surface is not significantly different from that of the native PFO monomers, otherwise the PFO monomers would preferentially interact with each other before interacting with $\mathrm{PFO}^{\mathrm{R} 468 \mathrm{~A}}$, which would have resulted in a less efficient conversion of the $\mathrm{PFO}^{\mathrm{R} 468 \mathrm{~A}}$ monomers to an inserted state.

\section{Structural features of cholesterol-bound ILY}

Our previous studies suggested that cholesterol binding by ILY was not necessary to trigger the D3 structural changes that are necessary for the formation of the oligomeric complex [32]: it appeared that CD59 binding, not cholesterol binding, initiated the D3 structural changes. Subsequent studies showed that the ILY CRM must initiate a cholesterol-dependent interaction to trigger the membrane insertion of loops L1-L3, which is necessary to anchor ILY to the membrane when it disengages from CD59 during prepore to pore conversion [33]. Therefore, if ILY could bind directly to cholesterol, in the absence of CD59, we predict that this interaction alone would not trigger the formation of the pore complex, as control of this process has been transferred to the CD59-binding site [30].

Although ILY does not bind significantly to cholesterol-rich cell membranes that lack human CD59 [29], we unexpectedly discovered that it binds well to cholesterol-rich liposomes, even better than PFO (Fig. 7A). Furthermore, this binding is dependent on the CRM, as a CRM knockout (ILY ${ }^{\mathrm{DM}}$ ) lacksdetectable binding to liposomes (Fig. 7A). Therefore, does this CRMmediated binding trigger the D3 structural changes like PFO and formation of a $\beta$-barrel pore? To address this question we first generated cholesterol-rich liposomes with entrapped 5(6)-carboxyfluorescein $(\mathrm{CF})$ and then treated them with PFO or ILY. The fluorescence emission of the concentrated liposome-trapped dye is quenched, but if the dye is released from the liposome its fluorescence emission increases upon dilution as it is released from the liposome $[39,40,41]$. PFO exhibited a dose-dependent release
A

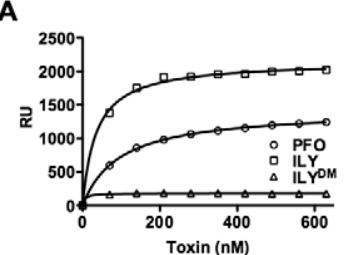

C

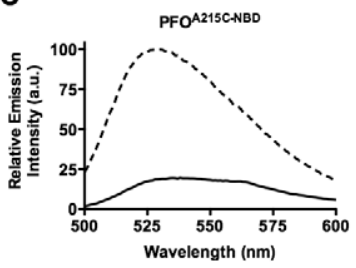

B
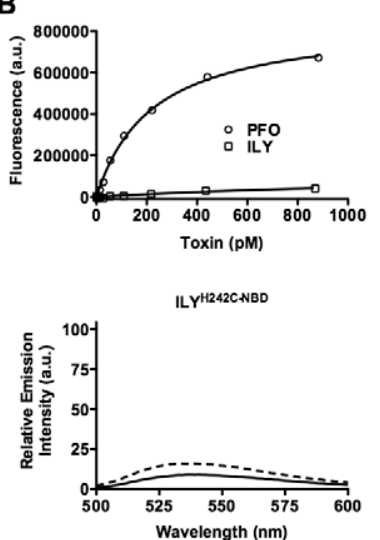

Figure 7. ILY binding and pore formation on cholesterol-rich liposomes. (A) Binding of PFO, ILY and ILYDM to cholesterol-rich POPC liposomes was measured by SPR. The data is representative of 3 experiments. (B) Pore formation on liposomes was measured as the emission intensity of CF increased upon dilution as pores are formed in the liposomes. The change in the emission intensity of CF over time in an untreated sample was subtracted from the experimental data. The data are representative of at least 3 analyses. ILYDM contains glycine substitutions for the ILY CRM residues Thr-517 and Leu-518, which knocks out CRM-dependent binding to cholesterol-rich membranes [22].(C) To measure the insertion of the $\beta$-barrel pore a cysteine was substitutedand modified with NBDfor TMH1 residue Ala-215 of PFO or its analog, His-242 in ILY. Each derivative was incubated in the presence (dashed line) and absence (solid line) of cholesterol-rich liposomes. As the soluble monomer binds to and forms a pore in the membrane the NBD probe positioned in $\mathrm{TMH} 1$ makes the transition from a polar environment in the soluble monomer (solid line) to the nonpolar environment of the membrane (dashed line), which is reflected by an increase in the NBD fluorescence emission [27]. doi:10.1371/journal.ppat.1002787.g007 
of the dye as evidenced by the increased emission of the dye as the concentration of PFO was increased. Although about twice as much ILY as PFO is bound to the liposomes, the ILY released less than $6 \%$ of dye released by $\mathrm{PFO}$ at the highest concentrations (Fig. 7B). We confirmed that the $\beta$-barrel of ILY was not inserting by measuring the insertion of TMH1. A NBD probe was position in TMH1 at cysteine-substituted His-242, which is a membrane facing residue in the $\beta$-barrel [42].Consistent with the lack of pore formation, ILY did not insert its $\beta$-barrel into the liposomal membranes (Fig. 7C).

Although pores were not forming, it was possible that the D3 $\beta 4-\beta 5$ interactionwas disrupted upon ILY binding to cholesterolrich liposomes. The disruption of the $\beta 4-\beta 5$ interaction is detected by a decrease in the emission intensity of an NBD probe positioned in $\beta 4$ as $\beta 5$ rotates away from $\beta 4$ its exposes the probe to the aqueous milieu thereby quenching its emission. This transition did not occur in the liposome bound ILY (Fig. 8), although it does occur in ILY bound to human CD59 containing cell membranes [32].Collectively these results suggest that while ILY can bind to cholesterol-rich POPC liposomes, like $\mathrm{PFO}^{\mathrm{R} 468 \mathrm{~A}}$ its binding does not trigger the D3 structural transitions necessary to initiate the formation of the oligomeric pore complex.

\section{Discussion}

The PFO pore forming mechanism is highly sensitive to changes in the undecapeptide structure $[6,13,16]$, but until now the molecular basis for its role in the CDC mechanism has been elusive. The studies herein show that mutation of the undecapeptide arginine residue uncouples membrane binding from the D3 structural transitions, which are necessary for the assembly of the pore complex.This mutation blocks all detectable structural changes in D3and prevents the stable interaction of the membrane-bound monomers. In essence, the structure of membrane bound monomers of this mutant appears relatively unchanged from that of the soluble monomer. Hence, for the first time these studies demonstrate a function for the conserved undecapeptide, which forms a critical structural elementin the allosteric pathway that couples membrane binding to the D3 structural changes that lead to pore formation. The studies also show that binding initiates changes through this allosteric pathway that allow monomer-monomer interaction, but it is the monomermonomer interactions that subsequently drive the major D3 structural transitions that are required for formation of the oligomeric pore complex.Furthermore, these studies show that this pathway has been lost in a CD59-binding CDC, which was a
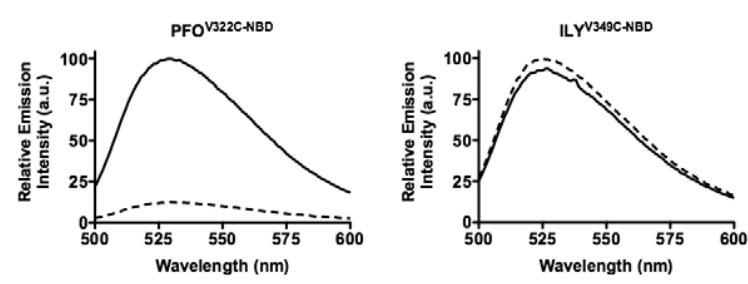

Figure 8. The $\beta 4-\beta 5$ interaction in cholesterol bound ILY. Cysteines were substituted for Val-322 in $\beta 4$ of PFO or the analogous Val-349 in $\beta 4$ of ILY and modified with NBD. Upon membrane binding $\beta 5$ rotates away from $\beta 4$, thus, the probe makes a transition from a nonpolar environment in the soluble monomer (solid line) to a polar environment in the membrane oligomer (dashed line), which results in the quenching of the NBD fluorescence [25]. The data are representative of 3 experiments.

doi:10.1371/journal.ppat.1002787.g008 necessary evolutionary step towards transferring control of this process from the cholesterol-binding site to the CD59-binding site.

These studies show that mutation of Arg-468 disrupts the allosteric signal that couples binding to the D3 structural changes that lead to the formation of the oligomeric pore complex and that functional derivatives of $\mathrm{PFO}$ can drive the major structural changes in D3, which are necessary for membrane insertion of the TMHs of $\mathrm{PFO}^{\mathrm{R} 468 \mathrm{~A}}$. Hotze et al. showed that monomermonomer contact could drive the major structural transitions in D3 in a mutant of PFO that was trapped in a prepore complex. Here we show that PFO can drive these changes in $\mathrm{PFO}^{\mathrm{R} 468 \mathrm{~A}}$, which is trapped at a much earlier stage where the monomers cannot interact with each other. Our data suggest that membrane binding is allosterically coupled to structural changes in PFO, which facilitate monomer-monomer interaction, but alone this allosteric pathway does not drive the major D3 structural transitions (i.e., disengagement of D2-3 interface and the $\beta 4-\beta 5$ interaction): these changes are driven by the subsequent interaction of monomers.

Soluble monomers of PFO do not interact and form oligomers, even at the high concentrations required for crystallization $[7,28]$. Therefore, PFO membrane binding must initiate structural changes in the monomers that facilitate their interaction. The monomer-monomer interactions then drive the major conformational changes within domain 3, whichare required for the formation of the $\beta$-barrel pore [35]. In this way PFO ensures an efficient assembly of the oligomeric pore complex on the target membrane. Membrane-bound $\mathrm{PFO}^{\mathrm{R} 468 \mathrm{~A}}$ monomers did not appear to form interactions that were of sufficient duration to be detected by FRET. For FRET to occur the donor and acceptor fluorophores must be at a fixed distance for a time that is equal to or greater than the half-life of the donor fluorescence emission, which is approximately $4 \mathrm{nsec}$ for the Alexa-488 dye [38]. Therefore, the mutation of Arg-468 appears to prevent the changes in the monomer structure that allows monomers to initially interact and form stable contacts that then drive the D3 structural changes. This mutation results in a membrane-bound monomer that appears to retain the structure of the soluble monomer, which cannot form any detectable intermolecular interactions.

In the crystal structure of PFO the only contacts made by Arg468 are hydrogen bonds between the NH1 of its guanidinium group and the backbone carbonyls of the CRM Thr-Leu pair (Fig. 1). Therefore, its substitution with alanine only prevents the formation of these two hydrogen bonds. This contact is interesting because it hydrogen bonds with the CRM, and may help stabilize the CRM structure in PFO. Hence, this contact may explain why mutation or chemical modification of the undecapeptide affects binding, as well as assembly of the oligomeric pore complex of PFO $[6,13,16]$. We cannot know with certainty, however, that this contact is essential to the allosteric pathway, only that the substitution of Arg-468 disrupts the allosteric pathway that couples membrane binding to the formation of the pore complex. The crystal structures of the cholesterol-binding CDCs PFO [7], suilysin (SLY) [43] and anthrolysin O (ALO) [9] have revealed that the undecapeptide $3 \mathrm{D}$ structure is highly variable: no two undecapeptides 3D structures have been shown to be the same $[7,9,43]$, even though their undecapeptide primary structures are identical. It is possible that these differences are due to an inherent flexibility of the undecapeptide and/or crystal contacts that affect the structural arrangement of the undecapeptides in the crystals. Hence, the conformational coupling of binding to the D3 structural changes may proceed through different undecapeptide mediated contacts in the CDCs. Alternatively, if the structure of 
the undecapeptide is flexible, as is suggested by the crystal structures, then membrane binding may lock it into a specific conformation that transmits the allosteric signal to D3, which cannot be achieved in $\mathrm{PFO}^{\mathrm{A} 468 \mathrm{~A}}$.

Functional PFO can drive D3 conformational changes in $\mathrm{PFO}^{\mathrm{R} 468 \mathrm{~A}}$ and the membrane insertion of itsTMHs in chimeric oligomers comprised of both proteins. Therefore, $\mathrm{PFO}^{\mathrm{R} 468 \mathrm{~A}}$ is structurally competent to form a pore, but lacks the conformational signal that initiates the necessary changes in its structure that facilitate the formation of stable intermolecular contacts. The fact that native PFO can drive these structural changes in $\mathrm{PFO}^{\mathrm{R} 468 \mathrm{~A}}$ indicates that it can establish a sufficient number of contacts with the $\mathrm{PFO}^{\mathrm{R} 468 \mathrm{~A}}$ monomers to drive these conformational changes in the latter. No stable intermolecular interactions of the $\mathrm{PFO}^{\mathrm{R} 468 \mathrm{~A}}$ monomers alone were detected by FRET showing that they do not interact, or that the interactions are transient and only exist on a timescale that cannot be detected by FRET. The ability of functional $\mathrm{PFO}$ derivatives to interact with $\mathrm{PFO}^{\mathrm{R} 468 \mathrm{~A}}$ indicates that at least one of the surfaces of $\mathrm{PFO}^{\mathrm{R} 468 \mathrm{~A}}$ is accessible to the functional PFO derivatives, which allows the functional PFO derivatives to dock with $\mathrm{PFO}^{\mathrm{R} 468 \mathrm{~A}}$. This interaction allows the functional PFO derivatives to establish contact with andsubsequently drive the structural changes in $\mathrm{PFO}^{\mathrm{R} 468 \mathrm{~A}} \mathrm{D} 3$ that are necessary for the formation of the oligomeric pore complex.

It is clear that $\mathrm{PFO}^{\mathrm{R} 468 \mathrm{~A}}$ bindingwas also affected by the Arg-468 to alanine mutation. If Arg-468 does make contact with the CRM carbonyls, as suggested by the PFO crystal structure, then it is possible that this substitution partially destabilized the CRM structure thereby affected binding. However, avidity may be a more important factor that contributes tothe difference in binding of wildtype $\mathrm{PFO}$ and $\mathrm{PFO}^{\mathrm{R} 468 \mathrm{~A}}$. Wildtype $\mathrm{PFO}$ and all mutants thereof generated to date still form membrane oligomers (most are represented herein). Oligomerizationis an important component of the binding interaction due to the avidity of the oligomeric complex versus the binding affinity of a single monomer. Oligomerization of PFO begins shortlyafter binding [37,44], thus binding of wildtype PFO and its derivatives reflects the avidity of the oligomeric complex rather than the affinity of single monomer.PFO ${ }^{\mathrm{R} 468 \mathrm{~A}}$ is the first mutant that has been described, whichis trapped in a monomer state on the membrane.Hence, therelatively poorbinding exhibited by $\mathrm{PFO}^{\mathrm{R} 468 \mathrm{~A}}$ may actually reflect the true binding interaction of native $\mathrm{PFO}$ monomers in the absence of oligomerization. It is also important to note that in the experiments in which functional $\mathrm{PFO}$ was used to drive the structural transitions in $\mathrm{PFO}^{\mathrm{R} 468 \mathrm{~A}}$ that we obtained near quantitative conversion of these transitions with a $4: 1$ molar ratio of functional $\mathrm{PFO}$ to $\mathrm{PFO}^{\mathrm{R} 468 \mathrm{~A}}$. If $\mathrm{PFO}^{\mathrm{R} 468 \mathrm{~A}}$ monomers bound the membrane with a significantly lower affinity than native $\mathrm{PFO}$ monomers then the probability of the native $\mathrm{PFO}$ monomers interacting with the $\mathrm{PFO}^{\mathrm{R} 468 \mathrm{~A}}$ monomers would be decreased and therefore it would be unlikely that we would have observed the near quantitative conversion of the $\mathrm{PFO}^{\mathrm{R} 468 \mathrm{~A}}$ monomers to a membrane-embedded state.

The CD59-binding CDCs, ILY [29], vaginolysin (VLY) [31] and lectinolysin (LLY) [45,46] exhibit undecapeptides with significant changes to their primary structures, most notably a proline substitution for the second conserved tryptophan (consensus, ECTGLAWEWWR;ILY, GATGLAWEPWR; VLY, EKTGLVWEPWW; LLY, EKTG $\overline{L V} W E \underline{W} W R)$. Unlike PFO, ILY does not maintain the hydrogen bond contacts between Arg-495 and the CRM (Fig. 1). This may be one of contacts in the cholesterol-dependent allosteric pathway that was disrupted during the evolution of the CD59-binding site, which was necessary to transfer of control of the D3 structural changes from the cholesterol-binding site to the CD59-binding site. Consistent with this scenario is the observation that substitution of the analogous arginine residue in ILY has little effect on the ILY poreforming mechanism [6]. We have shown herein that when ILY binds to cholesterol in the absence of CD59 it remains largely inert on the membrane, similar to what we observed for $\mathrm{PFO}^{\mathrm{R} 468 \mathrm{~A}}$. These data suggest that through evolution ILY has lost the allosteric pathway that couples cholesterol binding to the D3 structural changes in order to transfer control of the assembly of the oligomeric complex to the CD59-binding site [33,42,46].

Recently others have proposed that the membrane attack complex/perforin (MACPF) family of proteins may exhibit a CDC-like pore forming mechanism [47,48,49,50,51]. This proposal is based on the presence of a conserved protein fold that is similar to D3 of the CDCs [7], which we have shown forms the $\beta$ barrel pore structure of the CDCs [26,27]. The MACPF proteins play important roles in immune defense as well as in the pathogenesis of eukaryotic pathogens such as Toxoplasma gondii [52] and Plasmodium falciparum [53,54,55]. These proteins exhibit little sequence homology with the CDCs and do not exhibit an undecapeptide motif. It is possible, however, that they will alsoexhibit an analogous allosteric mechanism to regulate the assembly of their pore complex.

These studies provide the first evidence that shows the conserved undecapeptide plays an integral role in the allosteric coupling of cholesterol-mediated membrane binding to distal structural changes, which are necessary for the monomermonomer interactions that drive the assembly of the $\beta$-barrel pore.

\section{Materials and Methods}

\section{Bacterial strains, plasmids and chemicals}

The genes for native ILY and PFO were cloned into pTrcHisA (Invitrogen) as described previously [27,42]. All mutations were made in native ILY (naturally cysteine-less) or the cysteine-less PFO derivative $\left(\mathrm{PFO}^{\mathrm{C} 459 \mathrm{~A}}\right)$ backgrounds. The various CDCs and their derivatives are summarized in Table 2. All chemicals and enzymes were obtained from Sigma, VWR and Research Organics. All fluorescent probes were obtained from Molecular Probes (Invitrogen). Polyclonal anti-PFO antibody was affinity purified from hyperimmune rabbit serum. Secondary antibody goat anti-rabbit-HRP was obtained from BioRad. Sterols were obtained from Steraloids and lipids were obtained from Avanti Polar Lipids.

\section{Generation and purification of toxin derivatives}

PCR QuikChange mutagenesis (Stratagene) was used to make the various amino acid substitutions in native ILY or PFO ${ }^{\mathrm{C} 459 \mathrm{~A}}$ and DNA sequences of the PFO and ILY mutants were determined by the Oklahoma Medical Research Foundation Core DNA Sequencing Facility. The expression and purification of recombinant toxins and derivatives inEscherichia coliBL21 DE3 were carried out as previously described $[27,56]$. Purified protein was stored in HBS [100 nM NaCl, $50 \mathrm{mM}$ HEPES; (pH 7.5)], $50 \mu \mathrm{Mtris}(2$-carboxyethyl)phosphine (TCEP) and 10\% (vol/vol) glycerol at $-80^{\circ} \mathrm{C}$.

\section{Modification of cysteine-substituted toxin derivatives with fluorescent probes}

The labeling of PFO, PFO ${ }^{\mathrm{R} 468 \mathrm{~A}}$ and ILY cysteine-containing derivatives with IANBD [iodoacetamido-N, $\mathrm{N}^{\prime}$-dimethyl-N-)7-nitrobenz-2-oxa-1,3-diazolyl)ethylene-diamine; Molecular Probes] was carried out as previously described $[27,42]$. Toxin derivatives were labeled using a 20 -fold molar excess of the probe overnight at 
Table 2. PFO and its derivatives used herein.

\begin{tabular}{|c|c|}
\hline Toxin or its derivative & Description \\
\hline PFO & $\begin{array}{l}\text { Recombinant native PFO that contains a alanine substitution for the native cysteine (Cys-459) in the } \\
\text { undecapeptide [27] }\end{array}$ \\
\hline PFO ${ }^{\mathrm{R} 468 \mathrm{~A}}$ & PFO substituted at Arg- 468 with alanine \\
\hline $\mathrm{PFO}^{\beta 4 \beta 5}$ & $\begin{array}{l}\text { PFO with an engineered disulfide between } \beta \text {-strands } 4 \text { and } 5 \text { at cysteine substituted Thr- } 319 \text { in } \beta 4 \text { and Val- } 334 \text { in } \\
\beta 5\end{array}$ \\
\hline $\mathrm{PFO}^{\mathrm{R} 468 \mathrm{~A} \cdot(34 \beta 5)}$ & The analogous mutation to $\mathrm{PFO}^{\beta 4 \beta 5}$ \\
\hline $\mathrm{PFO}^{\mathrm{V} 322 \mathrm{C}}$ & $\begin{array}{l}\text { PFO with an engineered cysteine for Val-322. Val-322 is located in } \beta 4 \text { and is buried under the } \alpha 1 \beta 5 \text { loop. It } \\
\text { undergoes a nonpolar to polar transition as the } \alpha 1 \beta 5 \text { loop rotates away from } \beta 4 \text { [25] }\end{array}$ \\
\hline $\mathrm{PFO}^{\mathrm{R} 468 \mathrm{~A} \cdot \mathrm{V} 322 \mathrm{C}}$ & The analogous mutation toPFO ${ }^{\mathrm{V} 322 \mathrm{C}}$ \\
\hline $\mathrm{PFO}^{\mathrm{N} 197 \mathrm{C}}$ & $\begin{array}{l}\text { Cysteine-substituted Asn-197. Asn-197 undergoes a nonpolar to polar transition upon disruption of the D2-D3 } \\
\text { interface [27] }\end{array}$ \\
\hline PFO ${ }^{\mathrm{R} 468 \mathrm{~A} \cdot \mathrm{N} 197 \mathrm{C}}$ & The analogous mutation toPFO ${ }^{\mathrm{N} 197}$ \\
\hline $\mathrm{PFO}^{\mathrm{A} 215 \mathrm{C}}$ & Cysteine-substituted Ala-215 in TMH1. Ala-215 faces the membrane in the $\beta$-barrel pore [27] \\
\hline $\mathrm{PFO}^{\mathrm{R} 468 \mathrm{~A} \cdot \mathrm{A} 215 \mathrm{C}}$ & The analogous mutation to $\mathrm{PFO}^{\mathrm{A} 215 \mathrm{C}}$ \\
\hline ILY & Recombinant native ILY \\
\hline ILYDM & Cholesterol binding site knockout by glycine substitution for the Thr-Leu pair of the CRM in ILY \\
\hline ILYH242C & The analogous mutation in ILY to PFOA215C \\
\hline ILY & The analogous mutation in ILY to PFO ${ }^{\mathrm{V} 322 \mathrm{C}}$ \\
\hline
\end{tabular}

A summary of the CDCs and their derivatives used in the present study is shown.

doi:10.1371/journal.ppat.1002787.t002

room temperature $\left(22^{\circ} \mathrm{C}\right)$. The labeling reactions for the $\mathrm{PFO}^{\mathrm{V} 322 \mathrm{C}}$ derivatives also contained $3 \mathrm{M}$ guanidine hydrochloride to increase the efficiency of labeling.

Following the modification with the probes the mixtures were passed over a Sephadex G-50 column equilibrated in HBS. The labeled samples were made $10 \%$ (vol/vol)in glycerol and stored at $-80^{\circ} \mathrm{C}$. Proteins were typically labeled at an efficiency of $80-$ $100 \%$.

\section{Hemolytic activity of toxins and derivatives}

The cytolytic activity of the toxins and their derivatives on human red blood cells (hRBCs) was measured as previously described [27] except that the procedure was adapted to a microtiter plate format. Briefly, fresh human RBCs (hRBCs) were washed and suspended to $5 \%$ in phosphate buffered saline (PBS). The PFO and its derivatives were serially diluted in 2-fold steps in a microtiter plate at a final volume of $50 \mu \mathrm{l}$ per well to which $50 \mu \mathrm{l}$ of a $5 \%$ suspension of hRBCs was added and incubated for 1 hour at $37^{\circ} \mathrm{C}$. After incubation, unlysed RBCs were removed by centrifugation of the plate at $3400 \times \mathrm{g}$ for $10 \mathrm{~min}$. The $\mathrm{EC}_{50}$ for hemolysis (effective concentration of toxin for $50 \%$ hemolysis)was determined by quantifying hemoglobin release by measuring the absorbance of the supernatantat $540 \mathrm{~nm}$ using a DU640B spectrophotometer (Beckman).

\section{Liposome preparation}

Liposomes containing 1-palmitoyl-2-oleoyl-sn-glycero-3-phophocholine (POPC) and cholesterol at a ratio of $45: 55 \mathrm{~mol} \%$ were prepared as previously described [27]. Carboxyfluorescein-containing liposomes were made by adding $\mathrm{CF}$ [5(6)-carboxyfluorescein] to the cholesterol/lipid mixture in HBS at a concentration of $50 \mathrm{mM}$ before extruding [40]. After extrusion, the encapsulated liposomes were then passed over a Sephadex G-50 column in HBS pH 7.5 to separate unencapsulated CF from liposomes.

\section{Surface plasmon resonance analysis of ILY and PFO liposome binding}

Surface plasmon resonance (SPR) was performed with a BIAcore 3000 system (Oklahoma Medical Research Foundation) using an L1 sensor chip (Biacore) as previously described [22]. Binding analysis was performed as previously described [22] with the following modification: nine consecutive $10 \mu \mathrm{l}$ injections of the toxins and their derivatives (100 ng per injection) in HBS were passed over the liposome-coated chip at a flow rate of $10 \mu \mathrm{l} / \mathrm{min}$.

\section{Liposome release assay}

The pore forming activity of PFO and ILY and ILY DM was measured by incubating serial dilutions of toxin with $100 \mu \mathrm{l}$ of a 1:1000 dilution of carboxyfluorescein $(\mathrm{CF})$-containing liposomes in HBS for $1 \mathrm{~h}$ at $37^{\circ} \mathrm{C}$. Samples were read on a Victor3V Wallac 1420 Multilabel counter (Perkin Elmer) using wavelength settings optimized for high count fluorescein detection.

\section{Flow cytometry}

Two-fold serial dilutions of $\mathrm{PFO}^{\beta 4 \beta 5}$ and $\mathrm{PFO}^{\mathrm{R} 468 \mathrm{~A} \cdot \beta 4 \beta 5}$ labeled with Alexa-488 were incubated with washed human RBCs $\left(1 \times 10^{6}\right.$ cells) in PBS for $30 \mathrm{~min}$ at $4^{\circ} \mathrm{C}$.Samples were then brought to a final volume of $500 \mu \mathrm{l}$ with cold PBS and analyzed by a FACSCalibur flow cytometer (University of Oklahoma Health Sciences Center), gating on live cells. The emission wavelength was $530 \mathrm{~nm}$ and the excitation was $488 \mathrm{~nm}$ with a bandpass of $30 \mathrm{~nm}$. The disulfide locked $\beta 4 \beta 5$ versions of PFO and $\mathrm{PFO}^{\mathrm{R} 468 \mathrm{~A}}$ have cysteines substituted for residues Thr-319 and Val-334 [25], which forms a disulfide that prevents $\beta 5$ from rotating away from $\beta 4$. This disulfide prevents the lysis, but not binding to the RBCs during flow cytometry [25].

The geometric mean fluorescence of RBCs alone was subtracted from the experimental data for both PFO derivatives and the net fluorescence was graphed using GraphPad Prism. 


\section{SDS-agarose gel separation of PFO monomer and oligomers}

SDS-agarose gel electrophoresis was performed as previously described [36]. Briefly, samples were incubated with different concentrations of washed $\mathrm{hRBCs}$, for $30 \mathrm{~min}$ at $37^{\circ} \mathrm{C}$. Toxins were maintained at $440 \mathrm{nM}$ and the $\mathrm{hRBC}$ s concentrations ranged from $2.5 \times 10^{7} / \mathrm{ml}$ to $2.5 \times 10^{8} / \mathrm{ml}$ in a final volume of $40 \mu \mathrm{l}$. Samples were solubilized with SDS sample buffer and the complexes were analyzed on a $1.5 \%$ SDS-agarose gel $(100 \mathrm{~V}$, $120 \mathrm{~min}$ ) and then transferred to nitrocellulose membranes. Protein bands were identified using rabbit anti-PFO antibody followed by horseradish peroxidase tagged goat anti-rabbit secondary IgG. The bands were visualized using a chemiluminescent substrate (ECL Western Blotting Detection Reagents, Amersham/GE Healthcare) and autoradiography.

\section{Human erythrocyte ghost membrane preparation}

Human erythrocyte (hRBC) ghost membranes were prepared as previously described with some modifications [27,42]. After hypotonic lysis of the hRBCs for $15 \mathrm{~min}$ at $4^{\circ} \mathrm{C}$ in lysis buffer [5 mM sodium phosphate (monobasic), $\mathrm{pH} 7.5,1 \mathrm{mM}$ EDTA], cytoplasmic constituents were separated from the membranes by dialysis with $2 \mathrm{~L}$ of the lysis buffer by recirculation through a Vivaflow $2000.2 \mu \mathrm{m}$ PES cassette (Sartorius Stedim Biotech). Membrane protein content was quantified using the Bradford method (Bio-Rad Protein Assay, Bio-Rad Laboratories, Inc.) as previously described [27].

\section{Fluorescence spectroscopy}

All fluorescence intensity measurements were performed using a Fluorolog-3 Spectrofluorometer with the fluorescence software (Horiba JobinYvon). NBD measurements were made using the following settings: an excitation wavelength of $480 \mathrm{~nm}$ and an emission wavelength of $540 \mathrm{~nm}$ with a bandpass of $5 \mathrm{~nm}$. Emission intensity was scanned between 500 and $600 \mathrm{~nm}$ at a resolution of $1 \mathrm{~nm}$ with an integration time of $0.1 \mathrm{sec}$. In a typical experiment, labeled and unlabeled samples containing $10 \mu \mathrm{g}$ total

\section{References}

1. Alouf JE, Billington SJ, Jost BH (2005) Repertoire and general features of the cholesterol-dependent cytolysins. In: Alouf JE, Popoff MR, editors. Bacterial Toxins: A Comprehensive Sourcebook. 3rd edition. London: Academic Press.. pp. 643-658.

2. Calbo E, Garau J (2011) Factors affecting the development of systemic inflammatory response syndrome in pneumococcal infections. Curr Opin Infect Dis 24: 241-247.

3. Keyel PA, Heid ME, Salter RD (2011) Macrophage responses to bacterial toxins: a balance between activation and suppression. Immunol Res 50: 118123.

4. Mostowy S, Cossart P (2012) Virulence factors that modulate the cell biology of Listeria infection and the host response. Adv Immunol 113: 19-32.

5. Tweten RK, Caparon M (2005) Injectosomes of the Gram Positive Bacteria. In: Hultgren SJ, Caparon M, Waksman G, editors. Structural Basis of Bacterial Pathogenesis. Washington, D.C.: American Society of Microbiology. pp. 223240.

6. Polekhina G, Giddings KS, Tweten RK, Parker MW (2005) Insights into the action of the superfamily of cholesterol-dependent cytolysins from studies of intermedilysin. Proc Natl Acad Sci U S A 102: 600-605.

7. Rossjohn J, Feil SC, McKinstry WJ, Tweten RK, Parker MW (1997) Structure of a cholesterol-binding thiol-activated cytolysin and a model of its membrane form. Cell 89: 685-692.

8. Xu L, Huang B, Du H, Zhang XC, Xu J, et al. (2010) Crystal structure of cytotoxin protein suilysin from Streptococcus suis. Protein Cell 1: 96-105.

9. Bourdeau RW, Malito E, Chenal A, Bishop BL, Musch MW, et al. (2009) Cellular functions and X-ray structure of anthrolysin $\mathrm{O}$, a cholesteroldependent cytolysin secreted by Bacillus anthracis. J Biol Chem 284: 1464514656.

10. Saunders FK, Mitchell TJ, Walker JA, Andrew PW, Boulnois GJ (1989) Pneumolysin, the thiol-activated toxin of Streptococcus pneumoniae, does not require a thiol group for in vitro activity. Infect Immun 57: 2547-2552. toxin each were incubated with hRBC ghost membranes (equivalent to $300 \mu \mathrm{g}$ of membrane protein) or $20 \mu \mathrm{l}$ liposomes in HBS for $15 \mathrm{~min}$ at $37^{\circ} \mathrm{C}$ before taking spectral measurements. For all experiments the fluorescence intensity of the unlabeled samples was subtracted from that of the fluorescent probe-labeled samples in order to control for the intrinsic fluorescence of the sample in the absence of the probe.

\section{Fluorescence resonance energy transfer (FRET) measurements}

FRET analysis was performed as previously described [35] with the following changes. The PFO and $\mathrm{PFO}^{\mathrm{R} 468 \mathrm{~A}}$ derivatives were labeled with either Alexa Fluor 488 (donor, D) or Alexa Fluor 568 (acceptor, A).Parallel samples were prepared containing $10 \mu \mathrm{g}$ of D-labeled toxin mixed with a 4-fold molar excess of either Alabeled toxin or unlabeled $(\mathrm{U})$ toxin in a total volume of $2 \mathrm{ml}$. To correct for light scattering and direct excitation of the acceptor, a sample was prepared in parallel in which unlabeled PFO or $\mathrm{PFO}^{\mathrm{R} 468 \mathrm{~A}}(\mathrm{U})$ replaced the donor-labeled PFO to create the UA sample, therefore net DA = DA-UA. The samples were mixed in the presence of $\mathrm{hRBC}$ ghost membranes (equivalent to $300 \mu \mathrm{g}$ of membrane protein) for 15 minutes at $37^{\circ} \mathrm{C}$ and the donor emission intensity measured from $500 \mathrm{~nm}$ to $600 \mathrm{~nm}$. The donor emission intensity of samples in which unlabeled $\mathrm{PFO}$ derivatives replaced donor-labeled PFO derivatives was measured and subtracted from the donor-labeled samples to control for any intrinsic fluorescence of the toxin or direct excitation of the acceptor.

\section{Acknowledgements}

We would like to acknowledge the technical assistance of P. Parrish.

\section{Author Contributions}

Conceived and designed the experiments: KJD RKT. Performed the experiments: KJD. Analyzed the data: KJD RKT. Contributed reagents/ materials/analysis tools: KJD RKT. Wrote the paper: KJD RKT.

11. Pinkney M, Beachey E, Kehoe M (1989) The thiol-activated toxin streptolysin O does not require a thiol group for activity. Infect Immun 57: 2553-2558.

12. Michel E, Reich KA, Favier R, Berche P, Cossart P (1990) Attenuated mutants of the intracellular bacterium Listeria monocytogenes obtained by single amino acid substitutions in listeriolysin O. Mol Microbiol 4: 2167-2178.

13. Sekino-Suzuki N, Nakamura M, Mitsui KI, Ohno-Iwashita Y (1996) Contribution of individual tryptophan residues to the structure and activity of theta-toxin (perfringolysin o), a cholesterol-binding cytolysin. Eur J Biochem 241: 941-947.

14. Korchev YE, Bashford CL, Pederzolli C, Pasternak CA, Morgan PJ, et al. (1998) A Conserved Tryptophan In Pneumolysin Is a Determinant Of the Characteristics Of Channels Formed By Pneumolysin In Cells and Planar Lipid Bilayers. Biochem J 329: 571-577.

15. Billington SJ, Songer JG, Jost BH (2002) The variant undecapeptide sequence of the Arcanobacterium pyogenes haemolysin, pyolysin, is required for full cytolytic activity. Microbiology 148: 3947-3954.

16. Iwamoto M, Ohno-Iwashita Y, Ando S (1987) Role of the essential thiol group in the thiol-activated cytolysin from Clostridium perfringens. Eur J Biochem 167: 425-430.

17. Tweten RK (2005) The cholesterol-dependent cytolysins; a family of versatile pore-forming toxins. Infect Immun 73: 6199-6209.

18. Nakamura M, Sekino N, Iwamoto M, Ohno-Iwashita Y (1995) Interaction of theta-toxin (perfringolysin O), a cholesterol-binding cytolysin, with liposomal membranes: change in the aromatic side chains upon binding and insertion. Biochemistry 34: 6513-6520.

19. Ramachandran R, Heuck AP, Tweten RK, Johnson AE (2002) Structural insights into the membrane-anchoring mechanism of a cholesterol-dependent cytolysin. Nat Struct Biol 9: 823-827.

20. Heuck AP, Hotze E, Tweten RK, Johnson AE (2000) Mechanism of membrane insertion of a multimeric b-barrel protein: Perfringolysin $\mathrm{O}$ creates a pore using ordered and coupled conformational changes. Molec Cell 6: 1233-1242. 
21. Heuck AP, Tweten RK, Johnson AE (2003) Assembly and topography of the prepore complex in cholesterol-dependent cytolysins. J Biol Chem 278: 3121831225.

22. Farrand AJ, LaChapelle S, Hotze EM, Johnson AE, Tweten RK (2010) Only two amino acids are essential for cytolytic toxin recognition of cholesterol at the membrane surface. Proc Natl Acad Sci U S A 107: 4341-4346.

23. Czajkowsky DM, Hotze EM, Shao Z, Tweten RK (2004) Vertical collapse of a cytolysin prepore moves its transmembrane $\beta$-hairpins to the membrane. EMBO J 23: 3206-3215.

24. Ramachandran R, Tweten RK, Johnson AE (2005) The domains of a cholesterol-dependent cytolysin undergo a major FRET-detected rearrangement during pore formation. Proc Natl Acad Sci U S A 102: 7139-7144.

25. Ramachandran R, Tweten RK, Johnson AE (2004) Membrane-dependent conformational changes initiate cholesterol-dependent cytolysin oligomerization and intersubunit $\beta$-strand alignment. Nat Struct Mol Biol 11: 697-705.

26. Shatursky O, Heuck AP, Shepard LA, Rossjohn J, Parker MW, et al. (1999) The mechanism of membrane insertion for a cholesterol dependent cytolysin: A novel paradigm for pore-forming toxins. Cell 99: 293-299.

27. Shepard LA, Heuck AP, Hamman BD, Rossjohn J, Parker MW, et al. (1998) Identification of a membrane-spanning domain of the thiol-activated poreforming toxin Clostridium perfringens perfringolysin $\mathrm{O}$ : an $\alpha$-helical to $\beta$-sheet transition identified by fluorescence spectroscopy. Biochemistry 37: 1456314574.

28. Feil SC, Rossjohn J, Rohde K, Tweten RK, Parker MW (1996) Crystallization and preliminary X-ray analysis of a thiol-activated cytolysin. FEBS Lett 397: 290-292.

29. Giddings KS, Zhao J, Sims PJ, Tweten RK (2004) Human CD59 is a receptor for the cholesterol-dependent cytolysin intermedilysin. Nat Struct Mol Biol 11: 1173-1178.

30. Wickham SE, Hotze EM, Farrand AJ, Polekhina G, Nero TL, et al. (2011) Mapping the intermedilysin-human CD59 receptor interface reveals a deep correspondence with the binding site on CD59 for complement binding proteins C8alpha and C9. J Biol Chem 286: 20952-20962.

31. Gelber SE, Aguilar JL, Lewis KL, Ratner AJ (2008) Functional and phylogenetic characterization of Vaginolysin, the human-specific cytolysin from Gardnerella vaginalis. J Bacteriol 190: 3896-3903.

32. Soltani CE, Hotze EM, Johnson AE, Tweten RK (2007) Specific proteinmembrane contacts are required for prepore and pore assembly by a cholesteroldependent cytolysin. J Biol Chem 282: 15709-15716.

33. LaChapelle S, Tweten RK, Hotze EM (2009) Intermedilysin-receptor interactions during assembly of the pore complex: assembly intermediates increase host cell susceptibility to complement-mediated lysis. J Biol Chem 284: 12719-12726.

34. Nakamura M, Sekino-Suzuki N, Mitsui K, Ohno-Iwashita Y (1998) Contribution of tryptophan residues to the structural changes in perfringolysin $\mathrm{O}$ during interaction with liposomal membranes. J Biochem (Tokyo) 123: 1145-1155.

35. Hotze EM, Heuck AP, Czajkowsky DM, Shao Z, Johnson AE, et al. (2002) Monomer-monomer interactions drive the prepore to pore conversion of a betabarrel-forming cholesterol-dependent cytolysin. J Biol Chem 277: 11597-11605.

36. Shepard LA, Shatursky O, Johnson AE, Tweten RK (2000) The mechanism of assembly and insertion of the membrane complex of the cholesterol-dependent cytolysin perfringolysin O: Formation of a large prepore complex. Biochemistry 39: 10284-10293.

37. Harris RW, Sims PJ, Tweten RK (1991) Kinetic aspects of the aggregation of Clostridium perfringens theta toxin on erythrocyte membranes: A fluorescence energy transfer study. J Biol Chem 266: 6936-6941.

38. Haugland RP (2010) The Molecular Probes Handbook: A Guide to Fluorescent Probes and Labeling Technologies. 11th edition. Life Technologies. 1076 p.
39. Chen RF, Knutson JR (1988) Mechanism of fluorescence concentration quenching of carboxyfluorescein in liposomes: energy transfer to nonfluorescent dimers. Anal Biochem 172: 61-77.

40. Rex S, Schwarz G (1998) Quantitative studies on the melittin-induced leakage mechanism of lipid vesicles. Biochemistry 37: 2336-2345.

41. Schlesinger PH, Saito M (2006) The Bax pore in liposomes, Biophysics. Cell Death Differ 13: 1403-1408.

42. Giddings KS, Johnson AE, Tweten RK (2003) Redefining cholesterol's role in the mechanism of the cholesterol-dependent cytolysins. Proc Natl Acad Sci U S A 100: 11315-11320.

43. Xu L, Huang B, Du H, Zhang XC, Xu J, et al. (2010) Crystal structure of cytotoxin protein suilysin from Streptococcus suis. Protein Cell 1: 96-105.

44. Hotze EM, Wilson-Kubalek EM, Rossjohn J, Parker MW, Johnson AE, et al. (2001) Arresting pore formation of a cholesterol-dependent cytolysin by disulfide trapping synchronizes the insertion of the transmembrane beta-sheet from a prepore intermediate. J Biol Chem 276: 8261-8268.

45. Farrand S, Hotze E, Friese P, Hollingshead SK, Smith DF, et al. (2008) Characterization of a streptococcal cholesterol-dependent cytolysin with a lewis $\mathrm{y}$ and b specific lectin domain. Biochemistry 47: 7097-7107.

46. Wickham SE, Hotze EM, Farrand AJ, Polekhina G, Nero TL, et al. (2011) Mapping the Intermedilysin-Human CD59 Receptor Interface Reveals a Deep Correspondence with the Binding Site on CD59 for Complement Binding Proteins C8alpha and C9. J Biol Chem 286: 20952-20962.

47. Hadders MA, Beringer DX, Gros P (2007) Structure of C8alpha-MACPF reveals mechanism of membrane attack in complement immune defense. Science 317: 1552-1554.

48. Rosado CJ, Buckle AM, Law RH, Butcher RE, Kan WT, et al. (2007) A common fold mediates vertebrate defense and bacterial attack. Science 317: 1548-1551.

49. Slade DJ, Lovelace LL, Chruszcz M, Minor W, Lebioda L, et al. (2008) Crystal structure of the MACPF domain of human complement protein C8 alpha in complex with the C8 gamma subunit. J Mol Biol 379: 331-342.

50. Law RH, Lukoyanova N, Voskoboinik I, Caradoc-Davies TT, Baran K, et al. (2010) The structural basis for membrane binding and pore formation by lymphocyte perforin. Nature 468: 447-451.

51. Lovelace LL, Cooper CL, Sodetz JM, Lebioda L (2011) Structure of human C8 protein provides mechanistic insight into membrane pore formation by complement. J Biol Chem 286: 17585-17592.

52. Kafsack BF, Pena JD, Coppens I, Ravindran S, Boothroyd JC, et al. (2009) Rapid membrane disruption by a perforin-like protein facilitates parasite exit from host cells. Science 323: 530-533.

53. Kadota K, Ishino T, Matsuyama T, Chinzei Y, Yuda M (2004) Essential role of membrane-attack protein in malarial transmission to mosquito host. Proc Natl Acad Sci U S A 101: 16310-16315.

54. Kaiser K, Camargo N, Coppens I, Morrisey JM, Vaidya AB, et al. (2004) A member of a conserved Plasmodium protein family with membrane-attack complex/perforin (MACPF)-like domains localizes to the micronemes of sporozoites. Mol Biochem Parasitol 133: 15-26.

55. Ishino T, Chinzei Y, Yuda M (2005) A Plasmodium sporozoite protein with a membrane attack complex domain is required for breaching the liver sinusoidal cell layer prior to hepatocyte infection. Cell Microbiol 7: 199-208.

56. Soltani CE, Hotze EM, Johnson AE, Tweten RK (2007) Structural elements of the cholesterol-dependent cytolysins that are responsible for their cholesterolsensitive membrane interactions. Proc Natl Acad Sci U S A 104: 20226-20231.

57. Tilley SJ, Orlova EV, Gilbert RJ, Andrew PW, Saibil HR (2005) Structural basis of pore formation by the bacterial toxin pneumolysin. Cell 121: 247-256.

58. Humphrey W, Dalke A, Schulten K (1996) VMD: visual molecular dynamics. J Mol Graph 14: 33-38. 